\title{
MBAA Annual Conference Poster Abstracts
}

\section{Brewhouse Operations}

50. Urethane cements or epoxy toppings as flooring in a brewery. A. Llanes, International Coatings, Inc., Franklin Park, IL.

The focus of the presentation will be a detailed discussion pertaining to the use of urethane cements versus epoxy floor toppings in areas of high use, mechanical wear, and routine chemical and water wash down. It will also discuss the long history of successes and failures of some epoxy formulations and/or similar products and the rise of urethane cements over the past 10 years as a potential solution. The presentation will include detailed information on physical properties such as tensile and compressive strengths of both formulations and will also outline chemical and temperature resistance, wearability, slip resistance, and overall appearance and longevity. Also to be addressed are the basic issues dealing with surface preparation techniques, as well as a brief discussion on the most common concerns about moisture in concrete, its effect on proper installation, and long-term life cycle for both systems. Finally, the presentation will include input from installation contractors for both systems that includes ease of installation, workability, time factors for installation, installation techniques (slurry systems, troweled systems, top-coated or non-coated), and methods for repair in the event of compromise or failure. The presentation is an original work.

Angel Llanes, who has more than 30 years of experience in the field, initially as an installation contractor, then as a pioneer in rental and sales of surface preparation equipment, and now functioning as the vice president of sales for International Coatings Inc. Angel holds a B.S. degree in agriculture and M.S. degree in plant pathology from the University of Arizona.

51. Brewing with fermentable and non-fermentable carbohydrates addition-Impact on oxidative reactions and formation of specific aging compounds. N. O. Brandt, T. Kunz, and F. J. Methner, Technische Universität Berlin, Institute of Food Technology and Food Chemistry, Chair of Brewing Science, Berlin, Germany.

Carbohydrates are very important in human nutrition since they are used in the food and beverage industries. In this study the influence of fermentable and commonly used nonfermentable sugars on oxidative processes and the formation of specific aging compounds during beer production were evaluated. In previous investigations, the reducing potentials against $\mathrm{Fe}^{3+}$ of different sugars at low $\mathrm{pH}$ were determined using an optimized Chapon method. These results were in conflict with widely accepted general classifications of sugars into reducing and non-reducing species. The properties of carbohydrates at alkaline $\mathrm{pH}$ are well known, but in $\mathrm{pH}$ areas of wort and beer the properties of carbohydrates change depending on the type of sugar. For example, the so-called reducing

http://dx.doi.org/10.1094/TQ-50-4-1126-01

(C) 2013 Master Brewers Association of the Americas sugar glucose loses its reducing potential, whereby the reducing capacity of sucrose rises because of an acidic hydrolysis splitting and the strong reducing capacity of the generated fructose at low $\mathrm{pH}$ ranges. The aim of these investigations was to gain better insight into the influences of different carbohydrates during brewing, with a focus on oxidative wort and beer stability. Thereby, the influence of sugar additions on aging compounds (oxygen indicator: 3-/2-methylbutanal) were evaluated in wort and beer by GC-MS and compared to the antioxidative potential measured via EPR spectroscopy (EAP and T values), as well as reducing potentials against $\mathrm{Fe}^{3+}$ (optimized Chapon method) and $\mathrm{SO}_{2}$ contents. In correlation with the measured reduction potential, the sugars show the opposite effect on oxidative processes during wort boiling by the formation of specific intermediate Maillard reaction products with a reductone/endiol structure. An acceleration of radical generation by the Fenton-Haber-Weiss reaction mechanism and the formation of specific aging compounds follows. On the other side, sugars raise the osmotic pressure on yeast during fermentation, leading to higher sulfur dioxide production, which could act as an antioxidant by scavenging ROS and binding aldehydes in carbonyl complexes. The results show that sugars influence the pro- and antioxidative system of beer directly and lead to the suggestion for improvement of beer's shelf life by adding non-fermentable sugars just before fermentation, so the negative effect on radical generation during wort boiling can be avoided and the positive influence on $\mathrm{SO}_{2}$ formation during fermentation is utilized.

Niklas Brandt started his brewing career with an internship in a craft brewery in Lower Saxony, Germany. Afterward, he began an apprenticeship as a brewer and maltster at the Brauerei Beck \& Co. in Bremen (AB InBev Germany), which he successfully completed (2007-2009). Since October $2009 \mathrm{Ni}$ klas has been studying brewing and beverage technology at the Technische Universität Berlin. His bachelor degree study was finished in April 2013, and he is currently working on his master's degree studies. In addition to these studies, Niklas began research work in January 2011 as a student assistant at the Technische Universität Berlin, Institute of Food Technology and Food Chemistry, Chair of Brewing Science. It was here that his bachelor degree thesis work, dealing with research on sugars during brewing, was done. Niklas also works on the EPR spectrometer and assists with different projects in the laboratory and pilot brewery.

\section{Engineering}

52. Novel image cytometric method for detection of physiological and metabolic changes in Saccharomyces cerevisiae. L. L. Chan, A. Kury, and A. Wilkinson, Nexcelom Bioscience, Lawrence, MA; C. Berkes, Merrimack College, North Andover, MA; and A. Pirani, Nexcelom Bioscience, Lawrence, MA.

The study and monitoring of physiological and metabolic changes in Saccharomyces cerevisiae has been a key research area for the brewing, baking, and biofuels industries, which rely on these economically important yeasts to produce their products. Specifically for breweries, physiological and meta- 
bolic parameters such as viability, vitality, glycogen, neutral lipid, and trehalose content can be measured to better understand the status of $S$. cerevisiae during fermentation. Traditionally, these physiological and metabolic changes can be qualitatively observed using fluorescence microscopy or flow cytometry for quantitative analysis of fluorescently labeled cellular components associated with each parameter. However, both methods pose known challenges for the end-user. Specifically, conventional fluorescent microscopes lack automation and fluorescence analysis capabilities to quantitatively analyze large numbers of cells. Although flow cytometry is suitable for quantitative analysis of tens of thousands of fluorescently labeled cells, the instruments require a considerable amount of maintenance, highly trained technicians, and the system is relatively expensive to both purchase and maintain. In this work, we demonstrate the first use of Cellometer Vision for the kinetic detection and analysis of vitality, glycogen, neutral lipid, and trehalose content of $S$. cerevisiae. This method provides an important research tool for large and small breweries to study and monitor these physiological behaviors during production, which can improve fermentation conditions to produce consistent and higher quality products.

Leo Chan currently serves as the technology $R \& D$ manager and senior scientist at Nexcelom Bioscience LLC, Lawrence, $M A$. His research involves the development of instruments and applications for the Cellometer image cytometry system for detection and analysis of yeasts used in the brewing and biofuel industries. He is a member of MBAA. He received his B.S., M.S., and Ph.D. degrees in electrical and computer engineering from the University of Illinois at Urbana-Champaign (2000-2008).

53. Capital projects-From vision to completion. A. Hummer, ICC Inc., St. Louis, MO.

Since the early days of industrial brewing operations brewers have been faced with the challenges of converting their visions of capital projects into successful project completion. This is relevant for both expansion and replacement projects and also is relevant for small breweries to large mega plants. The challenge is multi-functional, and the variables are complex, such as in-house capability, previous project experience, project time versus cost, and relationship to third-party stakeholders, but also carry a great amount of subjective belief. The presentation will elaborate on a comparative analysis of various project execution methodologies and provide pros and cons for various scenarios. The presentation will also draw upon a global network of brewery capital project specialists in order to add a cultural element to the analysis from the five main continents where capital projects take place. The presentation will not make any specific recommendation but will bring audience members into a position where they can decide what will make sense for their individual needs. Finally, comparative analysis will show an academic approach to how the right methodology can be described as a function of internal competence and number of project units.

Anders Hummer, who is a partner of ICC Inc., draws on more than 20 years of international management and consulting experience and is educated in chemical engineering, master brewing, and business administration. Previous employment includes a partner position in Alfred Jorgensen Laboratory and international management positions at Danbrew. Anders also obtained management experience from Carlsberg Breweries and GlobalBrewer.

\section{Finishing and Stability}

54. Beverage antioxidative index (BAX)—An innovative method for the evaluation and improvement of the beer flavor stability. V. Schiwek, T. Kunz, and F. J. Methner, Technische Universität Berlin, Institute of Food Technology and Food Chemistry, Chair of Brewing Science, Berlin, Germany

For the prediction of beer flavor stability electron spin resonance (ESR) spectroscopy has been used for determination of lag time in the last decades. Previous investigations demonstrated that the lag-time measurement used up to now falsifies the results for oxidative flavor stability due to an increasing $\mathrm{pH}$ value during the analysis caused by the spin trap reagent $(\mathrm{PBN})$. Against this background, a new EAP determination was developed that excludes falsifications and offers a new index number for the evaluation of flavor stability: the beverage antioxidative index (BAX). As a complement to EAP determination, BAX provides additional information about the interplay of anti- and pro-oxidative beer ingredients independent of $\mathrm{SO}_{2}$ content and about the consumption rate of the antioxidative potential during storage. In this study, BAX determination was used to demonstrate the influence of metal ions, $\mathrm{pH}$, and Maillard reaction intermediates. Additionally, the correlation between BAX, beer color as an indicator of Maillard reaction products in the beer matrices, and formation of typical aging compounds during storage could be demonstrated. The investigations clearly demonstrate that lower $\mathrm{pH}$ values improve oxidative beer stability, as reflected by higher EAP, lower radical generation, and higher BAX values. Iron entry caused by raw materials and kieselguhr filtration deteriorate oxidative stability, and polyphenols do not change EAP and BAX significantly. Furthermore, it could be illustrated that hop ingredients like alpha- and beta-acids can act as a chelating agents and influence radical generation significantly and reduce oxidative processes. It could also be verified that specific intermediate Maillard reaction products with a reductone/endiol structure formed during kilning and wort boiling can decrease oxidative stability by the acceleration of the Fenton-Haber-Weiss reaction system and can have pro-oxidative properties. Corresponding to this, stronger generation of specific aging compounds (oxygen indicator) with higher color and lower BAX is detectable during beer aging. EAP determination facilitates a genuine examination of flavor stability. In combination with BAX determination, it is possible to get a deeper insight into the influences of different beer ingredients on flavor stability. An additional advantage of BAX is the indirect determination of $\mathrm{SO}_{2}$ content by linear regression.

Victoria Schiwek studied pharmaceutical and chemical engineering at the Beuth University of Applied Sciences from 2002 to 2007. The topic of her diploma thesis was "Optimized Analytical Methods for the Determination of $\mathrm{SO}_{2}$ in Beer and Malt." After graduating, she worked as a chartered engineer in the field of instrumental analysis, particularly HPLC and IC, at VLB-Berlin (2007-2009). Since 2010 she has worked as vice head of laboratory for the Research Institute of Brewing Science, Technische Universität Berlin (Berlin Institute of Technology).

55. Evaluation of pectin application as a stabilizing and fining agent for the brewing process. T. Kunz, G. Dingel, K. Rudolph, and F. J. Methner, Technische Universität Berlin, Institute of Food Technology and Food Chemistry, Chair of Brewing Science, Berlin, Germany. 
Fining agents are used to improve the filtration performance and reduce the production time of clear and bright beers, wines, and juices. Established fining agents like isinglass or gelatin are animal derived, and due to their allergenic potential, labeling is mandatory. In the literature pectin is described as a possible non-allergenic, plant-derived alternative to conventional fining agents. The aim of this study was to evaluate the application of pectin as a fining agent in the brewing process and to get a deeper insight into the mechanism of flocculation. Furthermore, the stabilizing effect of an aqueous pectin solution that contains sodium citrate, citric acid, and potassium meta-bisulfite was investigated to verify the possible suitability of pectin as a stabilizing agent as described in the literature. Lastly, a potential concept for the use of pectin was created. The results reveal that a pectin solution without additives does not increase the colloidal stability of beer by the removal of haze active polyphenol-protein compounds as described in the literature. Rather, the additives used, such as citric acid and particularly $\mathrm{SO}_{2}$, are responsible for a higher colloidal stability by increasing oxidative stability. Nevertheless, the suitability of pectin as a fining agent in the brewing process could be clearly verified. The efficiency of clarification depends on the pectin type used described by the degree of esterification, respectively amidation and the ratio of the beverage matrices $\left(\mathrm{pH}, \mathrm{AE}, \mathrm{Ca}^{2+}\right)$. Due to these complexities it is difficult to choose the right pectin for a new beer matrix without a testing method. For the creation of a potential concept for the pectin application in the brewing process and for the assessment of the fining suitability of pectins in a given beer matrix it was necessary to develop a laboratory quick test. Through the application of pectin two key factors of filtration, flow rate and filtrate haze, could be improved significantly. Lower filtrate haze did not lead to significantly better colloidal stability, but the possible reduction of filtration time by about $33 \%$ is emphasized. Residual galacturonic acid was not detectable (IC) in the final beer, which indicates that pectin is completely removed after filtration. With the right application of pectin in the brewing process and further optimization, pectin seems to be an efficient option to the established fining agents used in the brewing process.

After qualifying as a certified technician in preservation engineering (1991-1993), Thomas Kunz completed his basic studies in chemistry at the University of Applied Sciences, Isny (1994-1995), and his basic studies in food chemistry at Wuppertal University (1995-1998), before starting to study food technology at the University of Applied Sciences, Trier (19982002). After graduating, he worked as a chartered engineer in the area of ESR spectroscopy at the Institute of Bio Physics at Saarland University (2002-2004). Since 2005, he has been employed as a Ph.D. student at the Research Institute of Brewing Sciences, Berlin Institute of Technology (Technische Universität Berlin). His main research focus lies in analyzing radical reaction mechanisms in beer and other beverages using ESR spectroscopy.

56. Silica hydrogel removes gluten cross-reactive material from beer. K. A. Berg, PQ Corporation, Conshohocken, PA.

Three different beers were treated anaerobically in the laboratory with a range of doses of silica hydrogel. The beers were then analyzed for colloidal stability using a forcing test and the sensitive protein test provided by a tannometer. Unforced samples were also tested for gluten cross-reactive material using a simple dip stick test. The sticks were analyzed to collect quantitative information, which was then compared with the dose of the hydrogel and the colloidal stability results. Silica hydrogel quantitatively removes all gluten cross-reactive material at doses similar to those used to provide colloidal stability. Comparison with the sensitive protein test shows the sensitive protein measurement to not be specific for protein. Comparison to forcing results shows multiple beer changes due to the forcing itself. Colloidal stability is too complex to be adequately indicated by the gluten test.

Ken Berg received a B.A. degree in biology (biochemistry concentration) from Cornell University in 1976 and a Ph.D. degree in biochemistry from Brandeis University in 1981. After a postdoctoral appointment at NCSU, Ken designed protein purifications for Lee Scientific in St. Louis, MO. For the last 28 years he has aided $P Q$ Corporation by supporting its silica gel plants and their food industry customers globally. His customer-support techniques include biochemistry, microbiology, microscopy, and the chemistry of foods and silica. Ken lives near Philadelphia, PA, with his music teacher wife Shelley.

57. Inhibition of oxidative aging compounds in beer using active packing material vs. $\mathbf{S O}_{2}$-addition. C. Ruff, T. Kunz, and F. J. Methner, Technische Universität Berlin, Institute of Food Technology and Food Chemistry, Chair of Brewing Science, Berlin, Germany.

The origin of beer off flavors caused by oxidation has been attributed to the formation of specific aging compounds. The formation mechanisms, as different they are, mostly involve reactive oxygen species (ROS), highlighting the importance of intercepting oxygen reactions in the beer matrices or avoid oxygen entry during the brewing process and storage. The aim of this study was to figure out the different influences of oxygen entry and oxygen entry inhibition using active packaging material in the crown cap compared to the antioxidative activity of $\mathrm{SO}_{2}$ addition on the formation of aging indicators during storage. In this correlation also the combined application of active packaging material and $\mathrm{SO}_{2}$ addition on oxidative stability was investigated. Could the well-tried $\mathrm{SO}_{2}$ addition obtain the best results or is it possible to offer brewers new knowledge to improve beer flavor stability? Previous investigations have shown that techniques combining EPR spectroscopy and ascertainment of specific aging compounds as oxygen indicators via GC-MS can be used to investigate the influences of $\mathrm{SO}_{2}$ addition and different crown liners with $\mathrm{O}_{2}-$ scavenger properties on oxidative beer stability. The results after addition of oxygen to fresh beer samples verifies that the formation and increase in aging compounds is accelerated over storage time. In summary, the inhibition of oxidative processes by adequate $\mathrm{SO}_{2}$ addition in combination with after filling $\mathrm{O}_{2^{-}}$ scavenger material in the crown liner is the best way to increase oxidative beer stability during storage. However, the achievable increase in oxidative stability using different crown liner materials is strongly dependent on $\mathrm{O}_{2}$-scavenger properties. The compound with the lowest $\mathrm{O}_{2}$-scavenger properties showed an effect only after a longer storage time by the inhibition of oxygen diffusion through the crown cap and wasn't able to compensate for the advantage effect of $\mathrm{SO}_{2}$ addition at $6 \mathrm{mg} / \mathrm{L}$ on oxidative stability. The best $\mathrm{O}_{2}$ scavenger acted directly after filling, as well as during storage, and was able to compensate for $\mathrm{SO}_{2}$ addition at higher than $6 \mathrm{mg} / \mathrm{L}$. A remarkable and, for the mechanism, important point is that a comparable increase in oxidative stability with $\mathrm{SO}_{2}$ addition or $\mathrm{O}_{2}$ scavenger leads to a different positive effect on the formation 
of aging compounds during storage. In this connection with use of $\mathrm{O}_{2}$-active packing materials, a stronger reaction inhibition in the generation of specific aging compounds (oxygen indicators) was detectable. Altogether this research offers brewers useful further knowledge about how to proceed to increase oxidative beer stability through $\mathrm{SO}_{2}$ addition and/or active packaging material.

Constanze Ruff started her scientific career by participating in chemistry summer school at the Freie Universität Berlin before graduating. After school she interned for three months at Herbstreith \& Fox Company in Neuenbürg, Germany. She then began studies in chemistry at the Freie Universität Berlin. In March 2011 she switched to TU Berlin to the Department of Food Chemistry to complete her course of diploma studies in food chemistry. At the moment she is working on her prediploma (Studienarbeit). She began her research work in November 2010 as a student research assistant at the Technische Universität Berlin, Institute of Food Technology and Food Chemistry, Chair of Brewing Science, where she works on GC/MS detection of aging compounds and improvement of oxidative beer flavor stability.

58. Application of gallotannins to prevent gushing in beer and carbonated beverages. J. O. Schneidereit, T. Kunz, and F. J. Methner, Technische Universität Berlin, Berlin, Germany.

The spontaneous overfoaming of carbonated beverages upon opening a bottle without agitation is a problem that is wellknown in the beverage industry. Although the decoding of the occasion and mechanism of the "gushing effect" has been part of several research activities, a complete explanation has not been found yet. Numerous factors causing and contributing to gushing have been discussed. A first step toward solving this problem has been research to distinguish two types of gushing based on the origin of the gushing inducer. On the one hand there is malt-related gushing, known as primary gushing, which is caused by fungal metabolites. On the other hand secondary gushing is a consequence of technological faults during production. An important role in the formation of primary gushing is assigned to low molecular weight proteins, especially to fungus-specific hydrophobins. These proteins are secreted by various filamentous fungi. An important characteristic feature is the presence of eight conserved cysteine residues that build four intramolecular disulfide bridges. Furthermore, metallic ions like iron could be identified as an important influencing factor on gushing effect. Our previous investigations have shown that these kinds of metallic ions are involved in haze-active protein-polyphenol complexes and support or initiate the formation of equivalent complexes with protein-polyphenol compounds. The agglomeration of the described low molecular weight hydrophobin proteins that are co-complexed with these kinds of metallic ions are able to integrate and stabilize $\mathrm{CO}_{2}$ bubbles because of their high hydrophobin surface activity. Upon the release of pressure, the stabilized microbubbles, and in consequence the absorbed oversaturated $\mathrm{CO}_{2}$, expand and rise. Thereby, the bubbles entrain the surrounding liquid, resulting in overfoaming of the beverage. For the prevention of this process the responsible complexes of low molecular weight hydrophobin proteins, including metallic ions, need to be reduced or removed. An promising approach is reaction and complexation with gallotannins, which belong to the group of hydrolyzable tannins. Due to their chemical structure, gallotannins react primarily with $\mathrm{SH}$-group containing proteins like cysteine by adsorption and precipitation. Thereby, the emerging complexes, including the important gushing acting factors, can be removed nearly completely during the clarification steps. The gallotanninprotein complexes containing hydrophobin proteins and metallic ions are removed completely by whirl pooling, maturation, and filtration. Our studies have shown that the application of specific gallotannins in the right quantity and dosing point during the production or brewing process can be used to significantly reduce or avoid the gushing effect in beverages.

Jan Ole Schneidereit started his brewing career with an apprenticeship as a brewer and maltster at the Brauerei Beck and Co. in Bremen (AB InBev Germany), which he successful completed. Since October 2009, Jan Ole has been studying brewing and beverage technology at the Technical University Berlin. His bachelor degree studies were completed in June 2013, and he is currently working on his master's degree. In addition to these studies, Jan Ole began research work in November 2011 at the Technical University Berlin, Institute of Food Technology and Food Chemistry, Lab of Brewing Science. It was here that his bachelor's degree thesis work, dealing with the application of gallotannins to avoid gushing, was done. Jan Ole also works as a student assistant at the Technical University Berlin, Institute of Biotechnology, Laboratory of Microbiology.

59. Gallotannins-A useful tool to improve colloidal and oxidative beer stability. C. Reinhardt, T. Kunz, and F. J. Methner, Technische Universität Berlin, Institute of Food Technology and Food Chemistry, Chair of Brewing Science, Berlin, Germany.

In a highly competitive market place quality and consistency of beer have become of paramount importance. Besides beer flavor, color, and foam stability, one major attribute of beer quality is colloidal stability. If it comes to an undesirable haze during beer storage, the product is classified by the consumer as inedible. Although a variety of substances can be responsible for colloidal instability and haze formation, the most frequent and important form of non-biological haze formation has been identified as the interaction of proteins and polyphenols naturally occurring in beer. Our previous investigations have shown that metallic ions like iron with specific oxidation steps also have an important influence on the colloidal stability of beer caused by their influence on complex formation with protein-polyphenol compounds and oxidative stability. In order to obtain high colloidal product stability for a long period, it is necessary to remove the haze-active proteins and/or polyphenols using technological measures during the brewing process. Polyphenols are normally removed by nylon 66 or PVPP, while proteins can be removed with the help of silica gel or bentonite. In this correlation in the brewing industry it is increasingly popular to use gallotannins, as a high molecular weight tannic acid, to improve colloidal stability. When they are used in the process before filtration, they react primarily with haze-active acid SH-group containing proteins by adsorption and precipitation. The protein complexes that are formed are removed completely by filtration and are responsible for an increase in colloidal stability. Complexes that involve metallic ions like iron are also removed in this way. Consequently, a deceleration of oxygen activation and radical generation by the Fenton-Haber-Weiss reaction system is observable, resulting in a further advantage for colloidal and flavor stability as confirmed using EPR (EAP and T values), GC-MS (aging comp.), and other analytical methods. Our studies have shown that for 
the appropriate procedure (quantity, dosing point) the application of gallotannins is a good tool to increase oxidative and colloidal beer stability, resulting in lower formation of haze and typical aging compounds during storage.

After apprenticing as a brewer and maltster at the Braugold Brauerei Riebeck GmbH \& Co. KG Erfurt (2004-2007), Christof Reinhardt started his studies at the Technische Universität Berlin in biotechnology with a concentration in brewing technology (2008-2013). He began his research in January 2011 as a student research assistant at the Technische Universität Berlin, Institute of Food Technology and Food Chemistry, Chair of Brewing Science. He is working on determining the impact of fermentable and nonfermentable carbohydrates and gallotannins on the flavor and oxidative and colloidal stability of finished beer using EPR spectrometry.

\section{Food Safety}

60. Correlation of automated total $\mathrm{SO}_{2}$ DTNB method to EBC/ASBC para-rosaniline methods. M. Klemm, P. Ranta, L. Otama, and A. Suoniemi-Kähärä, Thermo Fisher Scientific, Vantaa, Finland.

Sulfur dioxide $\left(\mathrm{SO}_{2}\right)$ in beer primarily originates from yeast metabolism. $\mathrm{SO}_{2}$ reacts with carbonyl compounds to form hydroxysulfonates, which increase the flavor threshold of carbonyl compounds responsible for stale flavor. $\mathrm{SO}_{2}$ also has an important role as an antioxidant and is known to exert antimicrobial properties at high concentrations. $\mathrm{SO}_{2}$ level is controlled at the end of beer production due to human health and beer quality reasons. Total $\mathrm{SO}_{2}$ in beer typically is measured using EBC Method 9.25.3 or the similar ASBC Beer-21 prosaniline method. This paper shows the correlation of beer samples measured using the $p$-rosaniline method to the total $\mathrm{SO}_{2}$ method, which is based on DTNB (5,5'-dinitrobenzoic acid) measurement at $405 \mathrm{~nm}$. In this study, commercial beer samples and cider samples were analyzed using a Thermo Scientific Gallery discrete photometric analyzer. This rapid 2reagent Gallery system method was performed without any sample pretreatment before the analysis. A fully automated bench-top photometric analyzer concept enables simultaneous analysis of multiple parameters, like color, from the same sample. According to this study, all samples tested using the total $\mathrm{SO}_{2}$ method with Gallery system showed result levels similar to the EBC (9.25.3) and ASBC Beer-21 p-rosaniline methods. The easy-to-use Gallery system method is a robust method for total $\mathrm{SO}_{2}$ measurement for both cider and beer samples. Ready-to-use Thermo Scientific liquid reagents eliminate reagent preparation, saving time in testing, and the volume-optimized system kits minimize reagent waste. The method is linear from $2-50 \mathrm{mg} / \mathrm{L}$, and using automated dilution total $\mathrm{SO}_{2}$ can be measured up to $300 \mathrm{mg} / \mathrm{L}$.

Mari Klemm received an M.S. degree in analytical chemistry from the University of Helsinki in 2008. She joined Thermo Fisher Scientific in 2008 in their Research and Development Department, where she worked as an $R \& D$ scientist. Mari is a member of the $R \& D$ team responsible for development of important beer analyzing applications, like beta-glucan and NOPA, as well other food and environmental chemistry tests for discrete analyzers. She specializes in $\mathrm{SO}_{2}$ analytics, starting in 2007 with automating wine $\mathrm{SO}_{2}$ analysis. Since January 2013 she has functioned as the technology manager responsible for new industrial applications.

\section{Hops}

61. A review of the cost analysis of iso-octane regeneration for re-use in beer bitterness analysis. B. T. Bailey, Troegs Brewing Co., Hershey, PA.

The cost of purchasing and properly disposing of iso-octane (2,2,4-trimethylpentane) for testing bitterness in beer is considerable. It is by far the most expensive reagent used in the ASBC beer bitterness test. The method of regeneration described by Schild and Weyh in 1963 can regenerate iso-octane to the purity required for bitterness analysis in a modern brewery. Using their method of regeneration, savings can be realized almost immediately by any lab or brewery performing IBU analysis. In a lab that runs 10 bitterness tests/day following the ASBC method, a return on the investment in required equipment can be realized as quickly as eight months. When the costs of proper disposal of this hazardous chemical are considered, significant savings can be realized in less than eight months. By clarifying iso-octane through the column two times, it was possible to confirm that the IBU tests done with regenerated iso-octane are as accurate as those done with HPLC-grade iso-octane purchased from a chemical supplier. The beers tested during this trial ranged from 5.2 to $74.3 \mathrm{IBU}$, confirming the effectiveness of this method across a large range of beer styles.

Benjamin Bailey has been an MBAA member since 2010. His paper "The Influence of Hop Harvest Date on Hop Aroma in Dry-Hopped Beers" received the Inge Russell Best Paper Award in 2010. He received a B.A. degree in German from the University of Texas in Austin in 2000. After a three year apprenticeship at Live Oak Brewing Co. in Austin, TX, he enrolled at the University of Munich at Weihenstephan, graduating as a diploma master brewer in 2008. He then worked as a quality assurance engineer for MillerCoors in Trenton, $\mathrm{OH}$, before taking on his current role in 2010 as quality assurance team leader at Troegs Brewing Co. in Hershey, PA.

62. Comparison of Cascade hops grown in the U.S.A. and Germany. A. Gahr, Hopfenveredlung St. Johann GmbH \& Co. KG, Train-St. Johann, Germany; and A. Forster, HVG Hopfenverwertungsgenossenschaft e.G., Wolnzach, Germany.

Due to the huge demand, especially in craft brewing, more and more Cascade hops are grown in the United States and the high alpha market stagnates. So, other hop growing areas are looking for diversification. In 2012 German farmers harvested 6.1 tons of Cascade without hardly any knowledge of the comparability of their crop. The commonalities and differences of Cascade hops of different origin found in this work are presented. Cascade hops of U.S. and Hallertau origin were analyzed for bitter and especially aroma components, as well as for total and low molecular weight polyphenols, and used for all malt lager brewing trials. The single-variety brews focused on hop-derived sensory properties such as bitterness, late-hop, and dry-hop aroma. Analytical values were assessed and compared as well in order to obtain information about transfer rates of hop aroma compounds and polyphenols. Furthermore, some tests describe the losses of some individual aroma components during beer storage.

Andreas Gahr was trained on the job as a brewer and maltster at the Augustiner Brewery, Munich, Germany. He received a brewmaster degree from the Technical University MunichWeihenstephan in 1994 and worked for another four years at the university for the Chair of Brewing Technology I. Since 
1998 Andreas has been the head of the Research Brewery St. Johann, which belongs to the hop processing company Hopfenveredlung St. Johann GmbH \& Co. KG and deals with all kinds of hop-related brewing trials and product development, as well as technological and raw material trials for suppliers and the entire brewing industry. Together with his coauthors he received the MBAA Inge Russell Best Paper Award in 2010.

63. Use of microscopic pressurized shockwaves generated by controlled cavitation as a non-shear method for increased alpha-acid extraction in beer for later isomerization during the boil. S. Gomez, Apotek Solutions, Northville, MI.

Low yield in alpha-acid extraction during beer processing continues to be an issue in the brewing industry. Increased boil times do not necessarily translate to an increase in yield but do affect beer quality. Due to the sensitivity to shear of some beer compounds, standard food/beverage industry methods for mixing are not viable options. The use of microscopic shockwaves generated by induced cavitation is explored as a non-shearing mixing method for increased alpha-acid extraction prior to boil while maintaining specific hop characteristics (as opposed to using hop extracts). As cavitation bubbles form and collapse they generate intense pressurized shockwaves that travel through the liquid (water or wort) and hop resins increasing the surface area and mass transfer. A system specifically designed for this trial submits the liquid and hop cone/pellet mixture to cavitation and is subsequently boiled for $60 \mathrm{~min}$ for isomerization. The product was tested using the iso-octane extraction method and the iso-alpha method.

Santiago Gomez has been an MBAA Member (District Michigan) since 2012. Santiago has been a recognized BJCP judge since 2006, writer for the Michigan Beer Guide since 2011, and a home brewer since 2011. He has a B.S. degree in mechanical, electrical, and industrial engineering from Anahuac University in Mexico City and since 1993 has been involved in operation, design, engineering, and sales in the food and beverage industries. Santiago's primary areas of expertise include heat transfer and heat exchangers, mixing and homogenization, fermentation, enzyme treatments, extended shelf life, plant and equipment sanitation, pasteurization, and packaging. From 2001 to 2010 Santiago was responsible for the sale, design, engineering, manufacture, installation, and commissioning of food/beverage processing systems in countries such as the United States, Mexico, Canada, Colombia, Venezuela, Chile, Spain, the United Kingdom, Ireland, Belgium, The Netherlands, Italy, Russia, Thailand, and Taiwan, among others. Santiago currently works as an independent consultant in the food and beverage industries.

\section{Sensory}

65. It's all a matter of taste. R. Boughton, J. Gomez Lopez, and M. Pohler. FlavorActiV, Henley-On-Thames, U.K.

The United States is seeing explosive growth in beer styles and brands, which, in turn, has led to thousands more engaged in brewing, serving, and selling. Meanwhile hundreds of thousands more consumers are enjoying new beers and becoming enthusiasts wanting to learn more. Such a contrast has required new thinking on beer education, both in supply and consumption, as it is vital to maintain this wave of growth and choice. This paper will address how our global industry has and is responding to meet this challenge of educating our supply chain and reaching the consumer about taste.

Javier Gomez Lopez joined FlavorActiV in 2006 as a global sensory manager and covers the Spanish and Portuguese countries in Europe, Latin America, and Africa. He also looks after the craft brewers market in the United States and internationally. He has delivered many training courses in acceptance warehouse release, food and beer matching, train the trainer, sensory science and statistics. Javier is an expert in advanced and bespoke training courses, in addition to being FlavorAc$t i V$ 's in-house beer sommelier. Javier has recently played a key role in the development of web validation tools, making taster management quicker, easier, and more efficient. Javier has a Ph.D. degree in physical chemistry from Queen's University Belfast (2003); he worked as a research fellow at the university from 2003 to 2006.

\section{Sustainability}

66. Automated piping systems for increased product quality and reduced operating expenses in breweries. J. Hauser, Pentair Sudmo, Delavan, WI

Increasing product quality, which in turn increases shelf life, is a continuous goal for brewers. Piping systems used for beer transfer can have a negative effect on beer quality as it can introduce oxygen $\left(\mathrm{O}_{2}\right)$ through manual connections and open piping systems. This presentation will look at different valve options to prevent $\mathrm{O}_{2}$ pick up to increase plant efficiency and reduce operating expenses in breweries. It will look at advantages and disadvantages of current systems that are available and discuss an under tank matrix system that overcomes all of the disadvantages of the present systems. It will also discuss control-top technology for monitoring the valve system to ensure safe brewery operation. Swing panels were, and still are, used widely to route product and cleaning solutions through brewery piping systems and has the advantage of lower investment costs. However, operational costs are considerably more with it than an automated system because it is very operator dependent. It is also much more difficult to clean and to eliminate $\mathrm{O}_{2}$ pick up in a swing-bend system. The first matrix systems were standard manifolds that were positioned at the tank or in a central location. In recent years a new generation of piping system was introduced that is particularly suited to the brewing industry and eliminates many of the problems associated with the traditional valve matrixes, e.g., long pipelines between the vessel and the valve manifold are eliminated; as valves are close coupled to the vessel the problems of bacteria growth in the outlet pipes is eliminated; reduced space requirements; reduced investment costs; reduced $\mathrm{O}_{2}$ pick up; reduced product losses; and ease of installation with reduced costs. The matrix valves are used in conjunction with the IntelliTop control unit to ensure safe operation of the process. The IntelliTop monitors the valve position and gives continuous feedback to the control system for monitoring purposes. If the IntelliTop sends an alarm signal to the control system, the system will be set in a safe position to ensure the safety of product and personnel. There is also a clear visual signal from LED's that are fitted to the top of the control head that easily identifies the valve position or fault mode. During maintenance of the valve matrix a lockout feature is available to protect maintenance and operation personnel from contact with cleaning chemicals or other harmful liquids. The mix proof valve matrix system gives brewers the best possible piping option for their brewing vessels. Not only does it have lower 
investment and operating costs, it also ensures the highest quality product with the lowest possible $\mathrm{O}_{2}$ content, which will result in longer beer shelf life. Brewers around the world are embracing this technology as it gives them many benefits over conventional systems.

Jeremy Hauser, technical manager at Pentair Südmo, a supplier of process components and solutions to the brewing and beverage industries, has been involved in the automation of beverage and food plants for the past 13 years. He has been very active in advancing mix proof valve technology to increase plant efficiencies and reduce downtime. A member of $M B A A$, he attends regional meetings and training classes. He holds a bachelor's of mechanical engineering degree from the University of Illinois.

67. Coupled energy and water systems-The key to become a sustainable and self-sustaining brewer. D. F. Wagner, M\&L Engineering $\mathrm{GmbH}$, Hofheim am Taunus, Germany.

Sustainability is a major topic among brewers of the world. Large breweries strive to become "the greenest brewer." But, small and midsize brewers are interested as well, as sustainability combines economics and consumer decisive soft facts, like trust and credibility in a brand. In times of climate change and limitation of resources, self-sustaining brewers are less prone to taking risks. This is not only valid for developing countries, but for developed ones as well-just remember power outages, rising energy costs in grid dependent systems, and other influences that might result from changes in legislation and possible future market regulations. When analyzing the material and energy flows into the system brewery, it comes down to electric energy and one form of combustible primary energy on the energy side, as well as fresh water on the material flow side. On the out-feed side, the major flows are product (thankfully!) but even more waste water (figures!). All energy usage can be calculated as $\mathrm{CO}_{2}$ effluent, depending on the energy mix used. Under this precondition, a sustainable and self-sustaining brewer minimizes the in-feed of water and primary energy and the out-feed of wastewater and $\mathrm{CO}_{2}$ effluent, while being as grid independent as possible. With the CorEvapEnergy system, a brewer can make a tremendous leap to become more sustainable and self-sustaining. Especially for American brewers, with the dawn of widely available natural fracking gas this system provides electricity and heat energy at unbeaten efficiency levels, as well as being economically related to the carbon footprint. On the other side, it enables a reduction in freshwater consumption and amount of wastewater effluent—-truly a revolution! The CorEvapEnergy system consists of coupling natural gas-fired combined heat and electrical power generation with sophisticated evaporator technology. A highly efficient power generator transforms up to $43 \%$ of the primary energy into electricity. The residual thermal energy produced can be utilized either as process heat within the brewing process or to evaporate wastewater and recover the condensate as process water. Due to the continuous available heat sink of the evaporator, the electricity production can be continuously driven without the need to balance the energy streams of the brewery, even in non-continuous production phases. Process water recovery can be even increased by utilizing a vapor recompression unit to electrically drive the evaporation process. A total of up to $95 \%$ of the invested primary energy can be utilized.

After his apprenticeship in a small Bavarian brewery, Felix Wagner continued his education at the Technical University of
Munich-Weihenstephan, where he graduated as a diploma engineer in brewing. He started his professional career with KHS Till in 1999, where he was responsible for $R \& D$ in the field of kegging. During this time he concluded his doctorate at Weihenstephan. From 2005 he led the mechanical design team for KHS Process Technology in Bad Kreuznach, Germany, before moving to China to work for the KHS China Joint Venture as plant manager and deputy general manager. In 2010 he returned to Germany, taking the lead as head of the KHS Competence Centre for Filling and Kegging Technology. In 2012 Felix switched to $M \& L$ Engineering $G m b H$, which is focusing on evaporator technology, a part of the corosys group. corosys GmbH was founded in 2001 by partners with long-time experience in the field of process technology, automation, and instrumentation. Its focus is the production and supply of high-quality sensors, complete process systems and skids, components, and automation to the brewing industry.

68. Holistic heat storage system for breweries-Interface for renewable energy resources. $\mathrm{T}$. Becher and $\mathrm{K}$. Wasmuht, Ziemann International GmbH, Ludwigsburg, Germany.

Breweries are highly dependent on the environment for resources and, consequently, have a high level of environmental awareness. Therefore, an energy supply system is introduced that works using heat storage. It implies the change from steam-driven to hot water-driven operation. Utilization of fossil fuels is reduced and can be replaced by renewable energy resources. Natural sources such as solar thermic energy require a form of storage. The heat storage system functions with thermal stratification, which is advantageous for processes that require energy. The functionality of the system is explained by means of scientific survey, scenario simulation, and lead user experience (case study). How brewery equipment and technology can be adapted are described. With a holistic approach, the successful principle of energy recovery is extended and intelligently linked to all energy sources and sinks arising in the industrial operation. A functional interface for utilization of any available renewable energy is described.

Tobias Becher graduated as a diploma engineer of brewing science and beverage technology in 2001 from the Technical University of Munich in Freising-Weihenstephan (Germany). He apprenticed as a brewer and maltster before, worked afterward as a process engineer for beer filtration systems, and later worked as a technical consultant for environmental issues in the brewing sector. Since 2005 he has been employed by Ziemann. Today he is head of research and development within the Process Technology Division at Ziemann International GmbH (Germany).

\section{Utilities Management}

69. Optimizing brewing production with modular ondemand boiler systems. J. P. Smith, Miura North America, Inc., Atlanta, GA.

Boilers account for nearly half of industrial energy consumption and represent one of the most energy-intensive systems in the brewing industry. Breweries must manage production cycles that include highly variable heating loads that coincide with intermittent brewing, pasteurization, and CIP processes. Boiler energy management in brewery applications can often be constrained by the performance limitations of conventional, large-mass boilers that often exhibit overshoot and lag when operating in response to load swings resulting in 
a "tail wagging the dog" situation whereby the operation of the utility is driven by the capabilities of the boiler rather than by following sound energy management best practices. Tapping into years of experience with Japan's most well-known breweries, Miura has learned many lessons from lean manufacturing ("kaizen") principles that can be translated to the U.S. brewing industry to address waste related to overproduction via a "just in time" approach to process heating utilizing ondemand boiler systems, eliminating excessive energy consumption related to boiler idling, and stand-by losses during periods of reduced process loads. Much like tankless/instantaneous water heaters in residential applications, compact on-demand boiler systems offer increased energy efficiency (approximately 20-30\%) adapted to larger industrial applications. Given the large amount of energy consumed and the sharp minute-to-minute variations in process steam demands in the brewing industry, on-demand steam generation can play a significant role in reducing utility costs per unit of production, achieving the primary goal-more beer, fewer BTUs. These technology advances offer breweries significant benefits in their utility-side management, including reduced boiler footprint for optimized space utilization of utility versus production equipment; optimized energy management of variable loads during production; optimized back-up capacity with built-in $\mathrm{N}+1$ via modular design; flexibility to grow utility capacity with production via modular design; optimized staff utilization via on-demand operation and system automation; optimized boiler system O\&M via plug-and-play design; optimized safe operation due to minimal water content design; and reduced environmental impact from harmful emissions.

Jason Smith has a background in architecture and engineering with more than 15 years of experience with the design and construction of high-performance "green" buildings and more than 8 years of experience as an LEED Accredited Professional integrating sustainable design solutions into facilities that address energy efficiency and contribute to reducing their environmental impact. Jason is celebrating four years with Miura North America directing energy and environmental initiatives with a focus on energy efficiency advocacy and education in the area of thermal energy systems. Jason currently chairs the Energy Efficiency Deployment Sub-committee of the Department of Energy's ITP Steam Systems Best Practices Steering Committee and is an active member of the following organizations devoted to energy efficiency and sustainability: ESC, ACEEE, ASE, IDEA, APPA, ASHE, AEE, and USGBC. Jason is an active member of MBAA, having provided technical presentations for several MBAA Districts across the United States and Canada, as well as having presented at the 2012 World Brewing Congress.

70. Brew water treatment-Chemical reduced process. H. Fischer and F. Scheer, Krones Inc., Franklin, WI.

Water quality is one of the single most important factors for beverages. Making up over $80 \%$ of the product itself and having contact with the product during processing, there is an increasing focus on the microbiological quality of the water used in product processing. Since there is a formation of unwanted by-products when using chlorine as a disinfectant, one modern philosophy is a chlorine-free process.

Henri Fischer, brewmaster, has been the product manager of Process Technology at Krones, Inc., Franklin, WI, since 2013. Henri was team manager of the Process Technology 2 Division at Krones AG, Germany, until 2012. He worked in the Tech- nology Department of Steinecker, Germany, until 2006. Henri conducted his brewmaster studies at TUM Weihenstephan and Doemens Academy, Germany, finishing in 2003. He completed brewer and maltster training and was a brewer in a German brewery (Freiberger Brauhaus AG) from 1993 to 1998.

\section{Yeast, Fermentation, and Microbiology}

71. Standardizing cell concentration by weight method. A. Hammang, White Labs, Davis, CA; and T. Prahl, White Labs, San Diego, CA.

Yeast cell counts are an essential check on brewing operations, as fermentation is very sensitive to yeast concentration. Traditional cell counts under a microscope are a relatively simple and inexpensive method for validating yeast concentrations, but they are also time-consuming and cumbersome. Other cell counting methods are in use, each with their own down side for applications in the brewing industry: spectrophotometry (less accurate), plating (more time-consuming), flow cytometry (more expensive), and electrical resistance (more complicated operating procedures). The aim of this project is to validate and standardize a faster method for determining cell concentration that is still reasonably simple and inexpensive: cell concentration by weight. Cell concentration was determined by cell count in 70 yeast culture strains. Yeast cultures were then centrifuged and weighed in a test tube. A table has been constructed of the factor to correlate the weight of each strain to a cell concentration.

Annie Hammang has worked at White Labs' research and development lab for the past two years. She studied microbiology at the University of California at Davis and graduated in 2013 with a B.S. degree.

72. Use of spent brewer's yeast as a natural source of supplemental nitrogen in yeast propagations. N. Parker, White Labs, Inc., San Diego, CA.

Nitrogenous compounds, in the form of free amino nitrogen (FAN) or amino acids, are essential nutrients for proper yeast growth. These compounds are necessary building blocks for production of yeast biomass, including cellular proteins, as well as synthesis of critical enzymes required for metabolism and fermentation. Nitrogen supplements can be introduced to yeast as either amino acids or inorganic salts; however, typical inorganic ammonium such as diammonium phosphate, do not provide long-term benefits. Due to their more complex nature, mixtures of amino acids provide better yield and exert more positive effects, since they are also capable of providing a further carbon source during metabolism. Many commercial blends of assimilable nitrogen are available for both propagation and fermentation, but can spent brewer's yeast be used as a sole source of nitrogenous compounds? This presentation will discuss methods for extracting nitrogenous compounds from spent or discarded yeasts in the form of yeast extract, as well as a look at the free amino nitrogen content provided by yeast extract alone. The presentation will provide some potential methods for reprocessing spent brewer's yeast, as well as producing an affordable organic yeast nutrient source.

Neva Parker has been with White Labs, Inc. since 2002. She earned her B.S. degree in microbiology from Gonzaga University in Spokane, WA, and first became interested in the brewing industry while studying abroad in London. Neva currently manages laboratory operations and has been responsible for the development of new products and services, as well as re- 
searching the effects of various brewing aspects on yeast performance using lab-scale fermentation trials. She has presented at several workshops and conferences and published articles in brewing magazines. She is a member of MBAA and $A S B C$.

73. Practical application of $q P C R$ for monitoring and improving brewery sanitation. L. W. Fyfe and J. DeMerritt, Craft Brew Alliance, Portland, OR; D. Wedral, PALL, New York, NY; and D. Schleef, PALL, Portland, OR.

The growth of unpasteurized and unfiltered craft-style beers has increased the urgency around monitoring and improving brewery sanitation. The need for quicker methods, such as qPCR, provides an alternative method for detection of beerspoiling microorganisms before product is released into the marketplace. Traditional methods for detecting beer spoilers are limited and require a minimum of 5 to 7 days for results. Using quantitative PCR (qPCR) techniques, brewers can detect potential beer spoilers more quickly with higher sample throughput, as well as identify the type of spoiler they have detected. The authors will compare traditional plating methods with qPCR and describe how this information has been used to improve brewery sanitation in a large craft brewery. Actual examples from the brewery will be cited where traditional methods alone either were impractical or incapable of enumerating especially hard to detect bacteria, whereas the use of qPCR was able to identify the spoiler, as well as help identify opportunities for improved sanitation and new critical control points.

Leon Fyfe left academic research in 2011 and assumed the role of brewery microbiologist for the Craft Brew Alliance in Portland, OR. Leon's pursuit of a life in science started after an honorable discharge in 2005 from a 12 year career as a United States Marine. Leon graduated from Washington State University with a degree in biology and is currently a graduate student in the School of Molecular Biosciences. Leon has taken an active role in the MBAA Northwest District as a member of the Technical Committee. He continues his passion for high-quality craft beer through the spirit of education and collaboration.
74. Engineering a biosensor to detect a secreted yeast protease in beer. M. J. Farber, University of the Sciences, Philadelphia, PA.

Proteases are responsible for the cleavage and breakdown of other proteins. Previous studies have demonstrated that the yeast protease, proteinase A ( $\operatorname{PrA})$, also called saccharopepsin, is secreted in beer during fermentation. This protease hypothetically degrades foam-promoting proteins, thus decreasing head retention of the beer. Because the concentration of secreted PrA during fermentation has been correlated with yeast viability and proper nutrition, the precise measurement of PrA activity is important for quality control. Previous assays to measure PrA activity have demonstrated nonspecificity, low sensitivity, and high cost. Therefore, we have developed a novel, genetically encoded PrA sensor using engineered antibodies that fluoresce after cleavage by PrA. Using less than a drop of beer as the source of PrA, we can measure PrA activity over time. Thus far, we have demonstrated that mechanical disruption of yeast and prolonged storage of yeast increases the activity of PrA. In the future, we hope to use our PrA sensor to measure PrA activity induced by variables such as different yeast strains, improper handling of yeast, and prolonged back slopping. In addition, our platform will allow us to develop sensors for additional yeast proteases that might be relevant to the brewing industry.

Matthew Farber received a B.S. degree in biology from Seton Hall University (South Orange, NJ) and a Ph.D. degree in molecular and cellular biology from the University of Pittsburgh (Pittsburgh, PA). He is currently a postdoctoral fellow under Peter Berget at the University of the Sciences (Philadelphia, PA), specializing in cell biology and protein purification. Specifically, he engineers and produces biosensors capable of measuring the activity of proteases. His interest in brewing led him to apply these sensors to yeast targets relevant to the brewing industry. In addition to research, Matt teaches a graduate course in biotechnology and is currently developing the curriculum for an undergraduate course on the molecular biology of brewing. 